\title{
Implant Impression Making: Take-Off Guide for Beginners
}

\author{
Aaina Dhanda ${ }^{1}$ Tarun Kalra ${ }^{1}$ Manjit Kumar ${ }^{1} \quad$ Ajay Bansal $^{1}$ Ruchi Sharma ${ }^{1}$ \\ ${ }^{1}$ Department of Prosthodontics, Bhojia Dental College and Hospital, \\ Baddi, Himachal Pradesh, India \\ Dent J Adv Stud 2021;9:121-127. \\ Address for correspondence Manjit Kumar, MDS, Department of \\ Prosthodontics, Bhojia Dental College and Hospital, Baddi, 173205 , \\ Himachal Pradesh, India (e-mail: manjitkiran@yahoo.co.in).
}

\begin{abstract}
Keywords

- closed tray

- dental implant

- impression

- open tray

Dental implants are fixtures that constitute for the replacements of the root of a missing natural tooth. Dental implant therapy has been widely used for the restoration of partially and fully edentulous patients. The implant literature emphasizes the importance of a passively fitting prosthesis to prevent prosthodontic complications or even loss of fixture integration. Failure to achieve a passively fitting prosthesis and force tightening of superstructure may result in complications such as abutment, framework, and gold screw loosening or fracture. Various materials that can be used for making an implant impression are polyether, polyvinylsiloxane, condensation silicone, polysulfide, irreversible hydrocolloid material, and various others. There are various studies in relation to the accuracy of these impression materials out of which various scientists concluded different results with most studies stating polyether with the maximum amount of dimensional accuracy in comparison to other materials. An accurate implant impression plays a significant role and serves as a starting point in the process of producing good working casts. Thus, the accuracy of impression techniques becomes a significant issue in consideration of passive fit. Reproduction of intraoral relationship of implants through impression procedures is the first step in achieving accurate fit prosthesis. This transference is still complicated by the number, angulation, depth, and position of implants. The advent of computer-aided design/computerassisted manufacturing technology improved the framework fabrication procedures and has increased the precision of fit of implant prosthesis.
\end{abstract}

\section{Introduction}

Implant dentistry has empowered clinicians with new techniques to restore edentulous areas. There is an ever-expanding knowledge base regarding improved surgical and prosthetic protocols for implant reconstruction. The overall success is the ultimate replacement of the missing tooth; this can be best achieved through established prosthodontic principles. Whether or not the partially or fully edentulous condition is to be restored, it is important to understand all of the prosthetic steps necessary for treatment success. Proper impressions for implant dentistry are predicated upon conventional restorative techniques and therefore remain as one of the foundations for proper prosthetic reconstruction. ${ }^{1}$

To get accurate cast, impression accuracy is the key factor along with impression materials that plays a significant role published online November 10, 2021
DOI https://doi.org/

$10.1055 / \mathrm{s}-0041-1736489$. ISSN 2321-1482. (c) 2021. Bhojia Dental College and Hospital affiliated to Himachal Pradesh University. All rights reserved.

This is an open access article published by Thieme under the terms of the Creative Commons Attribution-NonDerivative-NonCommercial-License, permitting copying and reproduction so long as the original work is given appropriate credit. Contents may not be used for commercial purposes, or adapted, remixed, transformed or built upon. (https://creativecommons.org/ licenses/by-nc-nd/4.0/)

Thieme Medical and Scientific Publishers Pvt. Ltd., A-12, 2nd Floor, Sector 2, Noida-201301 UP, India 
in the same. ${ }^{2}$ There are various factors that affect the accuracy of the impression in implants, namely impression material, splinted and nonsplinted impression copings, type of splinted material, and number and angulation of the implants.

The materials used for making an implant impression are polyether, polyvinylsiloxane, condensation silicone, polysulfide, irreversible hydrocolloid material, and various others. Several authors have conducted studies about these impression materials out of which various scientists concluded different results with most studies stating polyether with the maximum amount of dimensional accuracy in comparison to other materials. Polyether impression material has least deviation from a custom fabricated master model. ${ }^{3}$ The type of tray also effects the accuracy of impression casts to multiple implants at different angles. In multiple implants at different angulations, type of tray used also plays a major role to get accurate cast. Open tray is better than closed tray for multiple implants at different angulation but according to some authors, ${ }^{4}$ the open tray technique was better suited for single implant than the close tray.

In recent years for the rehabilitation of both edentulous maxillae and mandible tilted implants are in practice. In mandible, the tilting of distal implants prevents injury to the mandibular nerve. There is increased distortion of the impression material during the removal from mouth due to presence of implant at different angles that may cause inaccurate cast. A study by Elshenawy et al in 2018 also concluded that the lesser angulated the implants, the least is the distortion. ${ }^{5}$ The splinting and nonsplinting impression copings are also a major factor that leads to accurate impression and cast. There are various materials that can be incorporated for splinting like floss, acrylic resin, stainless steel wire, and others. Many studies have been conducted to see the effect of direct techniques splinting and nonsplinting of the impression copings. A splinted coping with acrylic resin is better than unsplinted or splinting with light cure. ${ }^{6}$ Hence, splinting has better dimensional accuracy than the unsplinted technique. For better accuracy of multiple implant prosthesis, accuracy of impression is more important. Since the framework for multiple implants prosthesis has to be fabricated on the master cast, and if there is misfit, lots of stresses will be generated during fitting of framework on implants that leads to bone loss and detrimental to health of implants. ${ }^{7}$ For four or more implants, the pick-up impression technique is better than the closed-tray technique. ${ }^{8}$ There are mechanical and biological complications of misfit. Mechanically there will be screw loosening, implant fracture, and occlusal discrepancies. However, biologically this leads to increased plaque accumulation. To avoid future complications in implant prosthesis, misfit has to be minimized.

Certain precautions before impression making can be taken like taking a radiograph to confirm the level of seating of impression coping/abutment to the implant fixture, using a vinyl glove instead of a latex glove that retards the setting of the polyvinyl siloxane impression material.
Along with these precautions, there are certain postimpression laboratory steps that should be performed. After removal of the impression, it should be critically evaluated for any irregularity, porosity, etc. Disinfected implant analogues and impression copings are attached with each other and carefully relocated on the impression. Impressions should be washed to remove the saliva, blood debris, etc. and immersed in recommended disinfectant. Impression should be sent to the laboratory in a sealed box with proper work authorization.

\section{Factors Affecting Accuracy of Implant Impressions}

For fabrication of accurate master cast and passively fit framework, an accurate implant impression has a key role. There are numbers of factors that affect the accuracy of the impressions that can be listed as follows:

\section{Impression Technique}

Different impression techniques are indicated for different situation in implant prosthodontics. The techniques are broadly classified as the open-tray and the closed-tray technique. Among both the techniques, the open-tray technique exhibits greater dimensional accuracy and accurate linear distance measurements than the closed-tray technique. In case of single implant situation, however, the closed-tray technique demonstrates superior results. Many authors have studied repositioning of transfer copings that were difficult to place in their original position and become the source of big error in multiple implants. ${ }^{9}$

\section{Impression Materials}

When implant copings have undercuts, a material with good flexure strength is needed. The different properties of dental implant impression material like rigidity and dimensional stability influence the accuracy of the implant impression, solid implant cast, and implant framework. Various impression materials that are used in implant prosthodontics are:

- Hydrocolloid impression material

- Polyether impression material

- Polysiloxane impression material

All these materials have their own advantages and disadvantages. A study has been conducted to compare precision of these impression materials; polyether and addition silicone showed maximum dimensional stability and accuracy to the master casts. ${ }^{10}$

\section{Number of Implants}

Impression making for single implants is technically less demanding than multiple implants. For single tooth implant positional errors in the restorative stages are unlikely to affect passive fit with the implant, but rotational or dimensional discrepancy in the impression is likely to affect the appearance, contact points, and occlusal requirements.

The more the number of implants, the more are the chances for a dimensional inaccuracy to occur. Making 
impression of a single implant produces better results than a technique having to make impression of multiple implants. For multiple implants, the open-tray impression technique along with splinting showed better results than the other techniques.

\section{Angulation of Implants}

The implant angulation also affects the accuracy of the impressions. An implant that is placed at a 0-degree angulations poses lesser chances of distortion than the one which has implants placed at 15- or 30-degree angulation as in the posteriors. For angulated implants, addition silicone is the best material, and for parallel implants, polyether is the recommended material of choice. ${ }^{11}$

\section{Type of Tray Used}

The trays used in impression making in implants are the stock trays that are commercially available or the customfabricated trays according to the patient's mouth. Among both the trays, custom-fabricated trays provide better accuracy than the stock trays as they are prepared according to each individual.

\section{Splinting and Nonsplinting of Impression Copings}

For better prognosis of implant prosthesis, passive fit is the basic criteria. Impression accuracy is the key factor for accurate passive fit of the framework; otherwise, it might affect the health of the implants. Transfer of the precise position of implants to a master cast is a prerequisite for accurate and passive fit of the superstructure. Inaccurate frameworks can cause stress at the implant/bone interface. Although some previous investigations showed no difference in splinted or unsplinted pick-up impression copings, but on parallel sited implants. Among different splinting materials used that are the light cure, autopolymerizing acrylic resin, dental floss, pattern resin, it was found that splinting with acrylic resin demonstrates better results than the others. ${ }^{12}$

\section{Influence of Transfer Copings Surface Abrasion}

An impression transfer coping is used to place an implant analog in an impression and later on transfers to the master cast. Impression transfer copings are of two types:

An indirect transfer coping is screwed to the implant or the abutment body and remains in position after removal of impression from the mouth. It may be tapered or parallel sided for ease in removal (-Fig. 1).
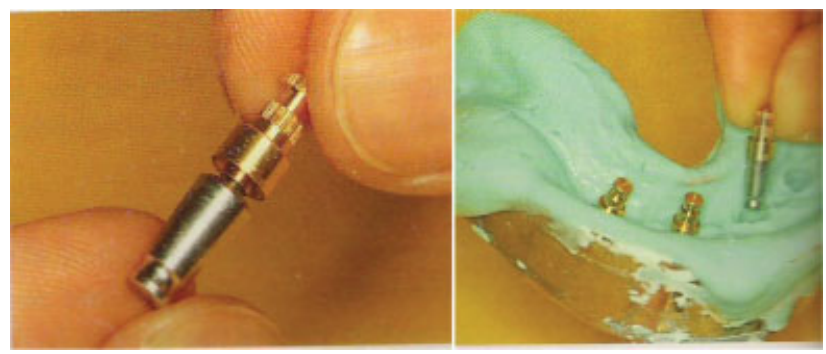

Fig 1 Indirect transfer copings.

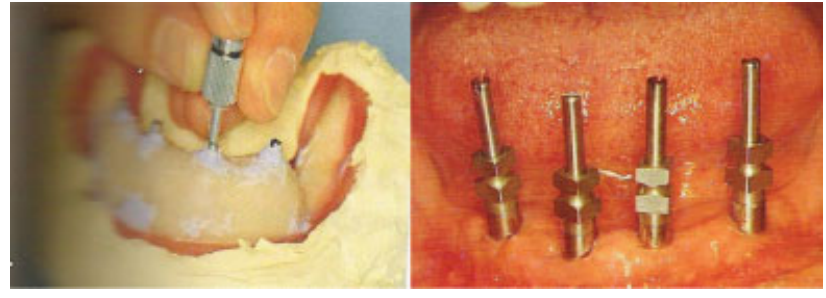

Fig 2 Direct transfer copings.

A direct transfer coping has a hollow transfer component with a long central screw to hold with the abutment or implant body and removed along with impression (-Fig. 2).

A square impression coping abraded with aluminum oxide gives better accuracy of impression and cast at an angle of 65 and 90 degrees. It is a simple and less timeconsuming method. In addition, working casts obtained from impression techniques using square impression copings splinted with autopolymerizing acrylic resin presented less inaccuracy in impressions with an implant inclination of 65 degrees. From this, it is cleared that in implants perpendicular to the surface (90 degrees), there will be less displacement of the transfer coping and more precise master casts than inclined implants. The adhesive coated copings provide better accuracy in case of single tooth-implant placement.

In other implant system impression copings for direct and indirect techniques are available along with plastic, press fit, and snap on caps (-Figs. 3 and $\mathbf{4}$ ).

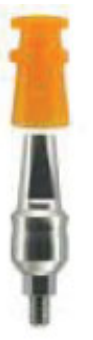

A
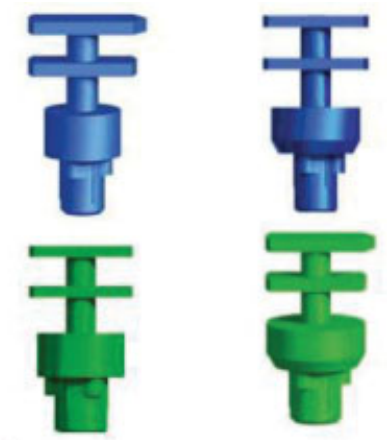

Fig 3 (A) Transfer coping with plastic cap. (B) Press fit impression copings.

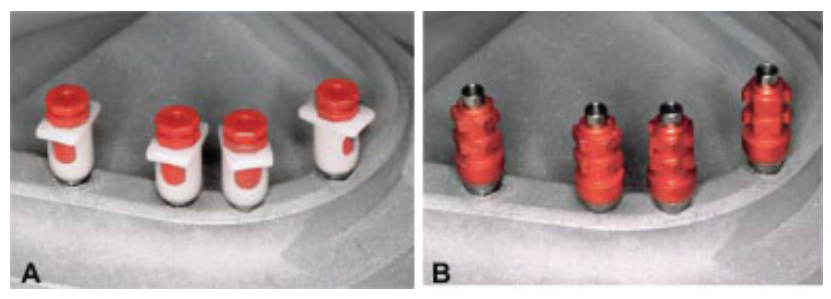

Fig 4 (A) Snap-on impression caps: Indirect impression technique. (B) Impression caps: direct impression technique. 


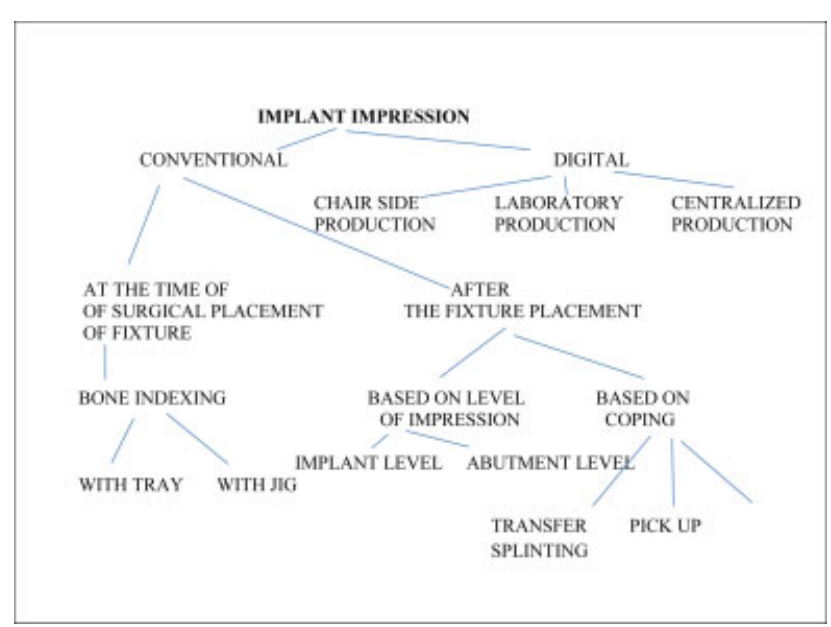

Fig 5 Classification of impression techniques in implants.

\section{Classification of Techniques}

The dilemma of implant prosthesis fit is the lack of an accurate clinical approach to measure misfit, which is further complicated by the difficulties of visualizing the implant and prosthesis interface. Therefore, clinicians and technicians should rely on controlling the precision of each step of implant prosthesis fabrication.

\section{Implant Impressions Classification}

The impression techniques for implants are broadly classified on the basis of:

- Type of tray used

- Type of technique used

- Material used
Implant impressions may be broadly classified as the open-tray and the closed-tray technique (-Fig. 5).

\section{Open-Tray Impression Technique}

In open-tray impression technique, implant position, hex orientation, and the soft tissue profile are transferred. In this technique, the healing screw is removed after 7 to 10 days of its placement. The transfer coping along with the abutment screw is threaded into the implant body. Custom tray is fabricated and special precaution is taken that the occlusal surface is open so that the abutment screw comes out through the opening. The custom tray has been adjusted so that the screw comes out through the opening. Impression is made with polyvinylsiloxane impression material. After the material has set, the dentist removes the abutment screw from the opening of the tray before removing the impression. After the screw is removed, the impression is removed. The transfer coping also comes out with impression and is embedded in the impression itself. Implant analog is attached to the impression post with the help of abutment screw before the impression is poured. Proper care is taken while threading the abutment screw to the implant analog that the transfer coping which is seated in the impression should not move. The impression is poured and working model is fabricated (-Fig. 6).

\section{Advantages}

In this technique, screws can easily be accessed and position of the transfer is also correct.

The main advantage of this technique is that the transfer coping comes out with the impression and less disturbances to the position transfers.

This technique is mainly used in nonparallel multiple implants in which the impression is easy to retrieve without distortion of impression material.

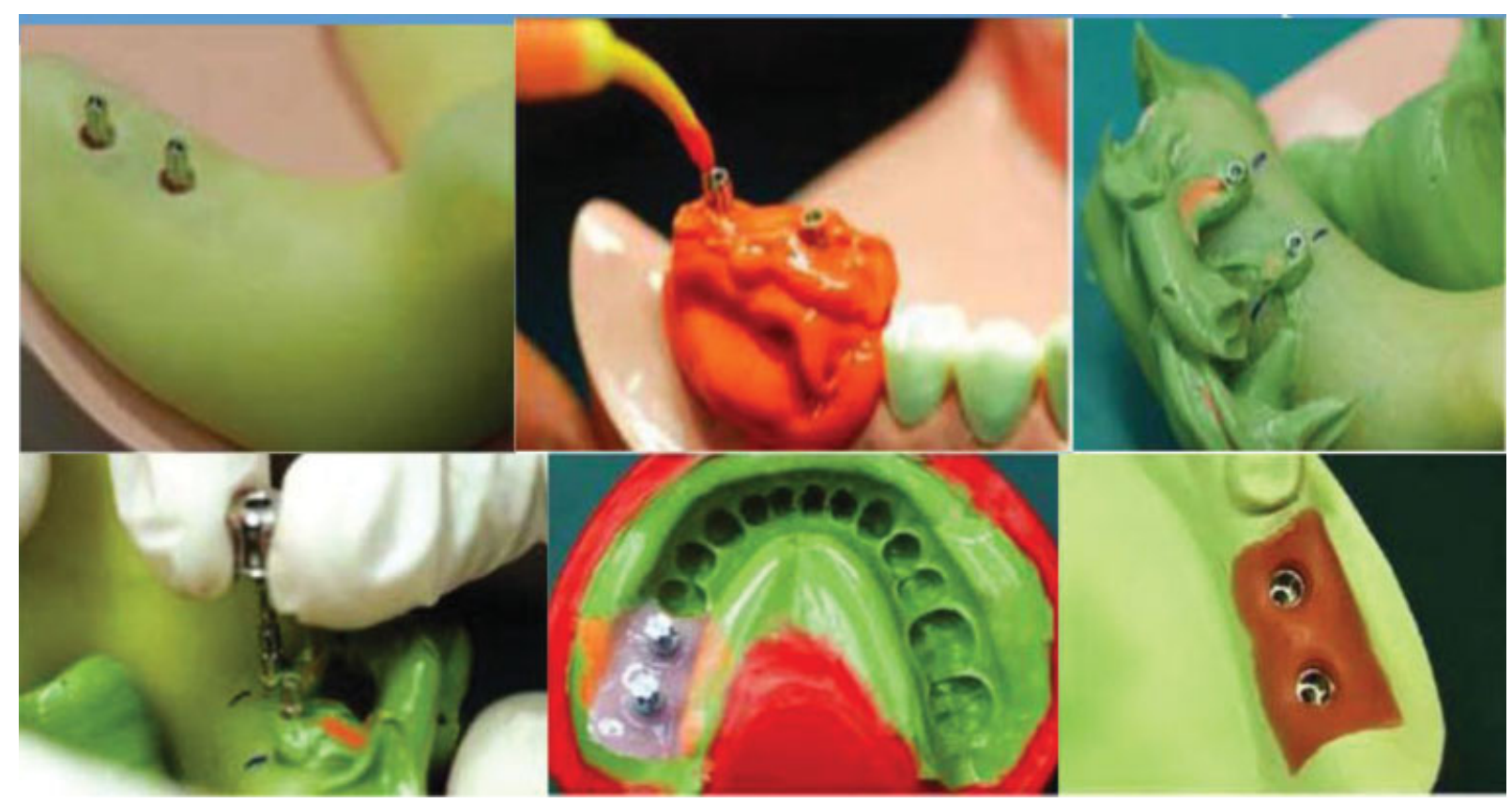

Fig 6 Open-tray impression technique. 


\section{Disadvantages}

There are more parts to manipulate. A custom tray with access to the impression coping screws is required or a metal tray with windows is needed.

Along with the type of technique used, the choice of the type of tray also greatly affects the accuracy of the impression making. In case of the open-tray impression technique, we can use custom trays as well as stock trays. A study suggested that the rigid custom trays are preferred over plastic stock trays. The impressions made with stock trays were less accurate. It was found that for analogs with $20 \mathrm{~mm}$ separation, there was a difference in $10 \mu \mathrm{m}$ in the accuracy between stock tray and custom trays. ${ }^{13}$

\section{Closed-Tray Impression Technique}

In the closed-tray impression technique, only the implant's position and hex orientation are transferred. When the impression is removed from the mouth, indirect transfers remain attached to the implants. The transfer copings are parallel sided or slightly tapered for easy removal of impression from the mouth. The impression is usually made after 7 to 10 days of placement of healing screw. Once the inflammation is reduced, the healing screw is removed and the transfer coping is screwed. A radiograph is taken to confirm the tight and perfect joint of the impression post and implant. The screw hole is blocked with the help of blocking wax to avoid the material to flow into the hole. The impression is made. As the material sets, the impression is removed from the patient's mouth, and the transfer coping remains in the patient's mouth. The dentist removes the transfer coping/impression post from the implant body, attaches it to the implant analogue, and then reinserts it into the desired position after proper orientation. Proper care has to be taken that the implant analogue along with the transfer coping should be properly oriented and inserted. Once the position has been finalized, the impression is poured and the working model is fabricated ( - Fig. $\mathbf{7}$ ).

\section{Advantages}

This technique is indicated in cases of limited mouth opening with hyper gag reflex.

\section{Disadvantages}

There might be coping dislodgement during impression removal. Abutments have to be fixed onto the copings, which may lead to an error at this stage. Soft tissue transfer is not very accurate and the size and shape of the abutment cannot be modified. The impressions removal is also not easy.

The type of transfer coping used in the closed-tray technique is usually tapered in shape and shorter than those used in the open-tray technique.

A study by Balouch et al in 2013 compared the accuracy of implants impression techniques in 15 degrees angulated implants and found that the closed-tray technique is better suited for making impression for angulated implants than the open tray technique. ${ }^{14}$

\section{Impression Technique for Completely Edentulous Patients}

In case of multiple abutments, the primary impression is made with the help of alginate impression material after 7 to 10 days of placement of abutment screws. The custom tray is fabricated on the primary cast after placement of suitable spacers. The occlusal surface of the tray is kept open so that the abutment screws can extend through the opening; after

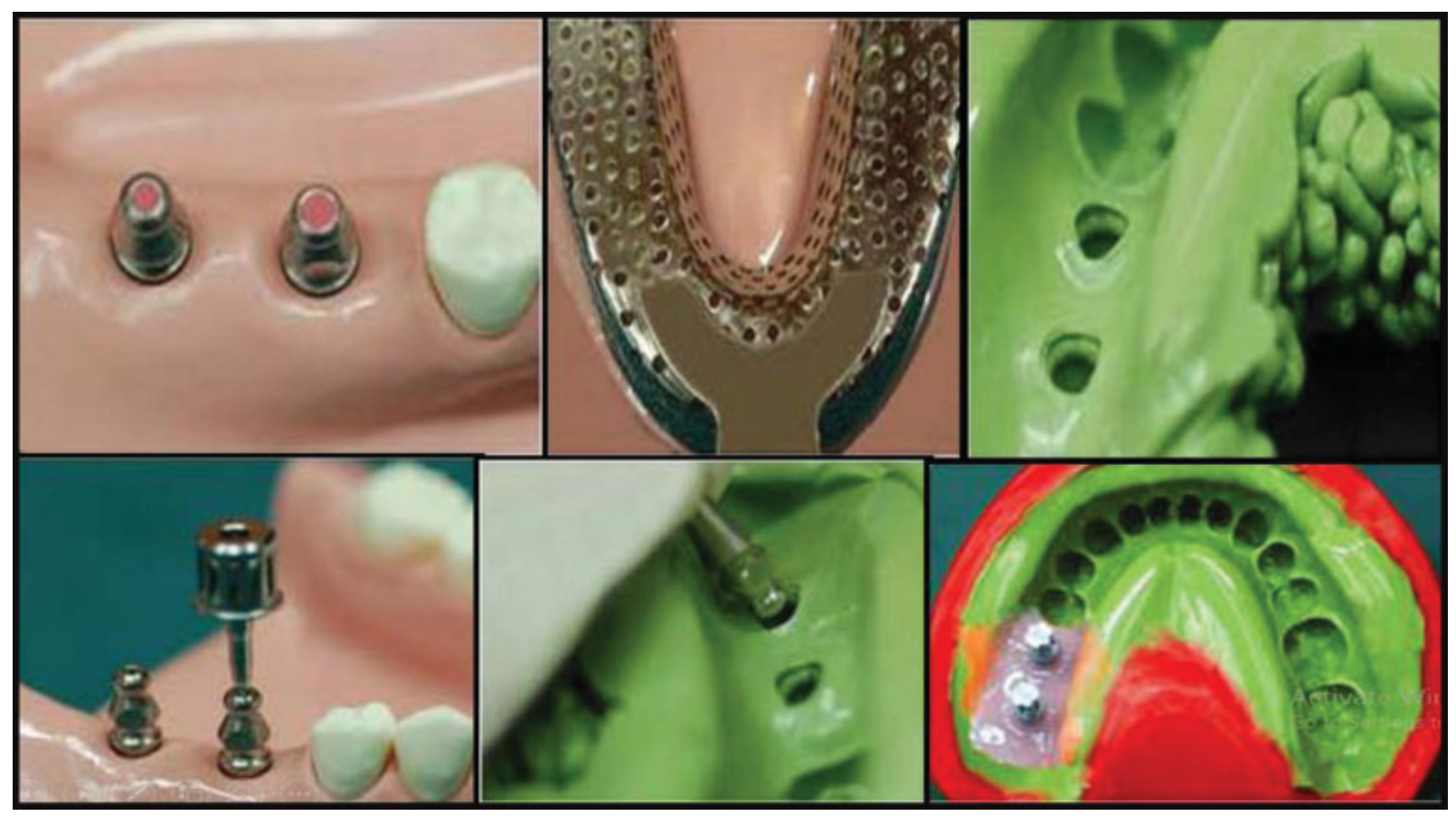

Fig 7 Closed-tray impression technique. 
the extensions of the tray is checked, the tray is placed in patient's mouth and the open surface is sealed with hard wax. This helps in orientation of the cast as well as prevents the flow of material from the opening. Impression of the arch is made with elastomeric impression material. The impression is poured and final cast is obtained. There is another method to make implant impressions of completely edentulous patients that is the splinted technique.

There are three parameters that are important, types of final impression method, that is, tissue-level, abutmentlevel, or implant-level impression; stage of overdenture fabrication, that is, record base stage, denture-processing stage, or denture insertion stage; and nature of technique, that is, direct or indirect. Square impression coping and the use of copings that have been airborne particle abraded and coated with impression adhesive show more accurate performance in replicating the reference resin models than the nonmodified technique or the unsplinted technique.

\section{Modifications of Impression Techniques}

Impression techniques can be modified based on different situations. The aim is to derive an accurate impression. In implant prosthodontics, impression making with open tray with a window is very common. It may be difficult for the guide pins to protrude from the opening of the wax lid, because the impression material in the tray can obscure the guide pins during the procedure. It has been experienced that if the tray is repositioned several times, the impression may be distorted and/or contains bubbles. If the opening of the wax lid is too wide, or if the wax lid is out of place, the pressure on impression tray may decrease, and the impression material may not extend over the soft tissue around the implants, especially in the maxilla. Incomplete soft tissue impression around implants hinders the fabrication of a superstructure with a proper emergence profile. A modified implant impression technique is presented as a solution to these problems.

Different modifications can be listed as follows:

\section{Functional Impression Technique}

An impression made for fabrication of overdenture must transfer the soft tissue profile as well as accurate location of the implant. An overdenture is just like a complete denture, only difference is dual support from implant and soft tissue. In implant-retained overdentures, there is difference in compressibility of mucosa and implant. So, the mucosa should be recorded in a functional state. The main advantage of this technique is recording of accurate relation of the implant and soft tissues. After insertion of the prosthesis, chair time decreases for postinsertion adjustments. However, the procedure is technique sensitive in recording of the border relation with different impression materials, and it is more time consuming compared with the single-stage impression. The functional impression technique records the mucosa in a functional state and it simultaneously records the implant components in relation to the alveolar tissues. In this technique, zinc oxide eugenol impression paste and elastomeric impression material are used.

\section{Tray-Less Impression Technique ${ }^{15}$}

A technique incorporating accuracy, simplicity, and speed is desirable when making complex impressions. This is called tray-less impression procedure, a technique not identified in other articles within the dental literature. Using this method, which was originally intended to facilitate impression making in the surgical field, the author has made complete arch impressions at the time of surgery for the fabrication of immediately loaded, single-piece, screw-retained provisional restorations supported by external hex implants. This technique is efficient and has also been used for making definitive impressions. This tray-less technique facilitates making impressions in edentulous patients with restricted access. Direct splinting has been shown to be the most accurate method for multiple abutment impressions. The prostheses fabricated using this impression technique are clinically and radiographically accurate, using accepted in vivo evaluation procedures.

Impression Techniques for Arches Requiring Both Implant and Natural Tooth Restorations

A modified impression technique is highly recommended where impression of the prepared tooth and implant have to be made together. Here, dimensional stability and fine details of the margins of the prepared tooth have to be recorded. When implant impression is made, fine details are not required as compared with prepared tooth. So, light body material can be injected around the prepared tooth with an impression syringe. When implant impression made with the impression coping close to the prepared tooth, the access will be less.

\section{Impression Technique for Implants Placed in Close Proximity or Adverse Angulations ${ }^{16}$}

For implants placed in close proximity, it becomes difficult to make an impression of the same; therefore, impression technique is used for making impressions in such situations. One coping is unmodified impression coping and the other is a modified impression coping with short fastening screw. Undercuts made on upper portion of shortened impression coping. Polyether and vinyl polysiloxane were the recommended materials for the implant impressions. The literature seems to be having inconsistencies in the acceptance of a superior technique.

\section{Recent Advances in Impression Making}

The use of digital impressions eliminates the need of impression materials, making the procedure potentially more comfortable for the patients while decreasing error from the analog techniques. ${ }^{17}$ With current computer-aided design/computer-assisted manufacturing (CAD/CAM) techniques, digital technologies once limited to scanning and milling of the tooth-supported fixed prosthesis can now support the implant impression procedures (-Fig. 8).

Direct intra oral scanning of the implant cannot only create a three dimensional (3D) virtual model to design and mill the restoration but can also be used to fabricate a milled model with a removable implant analogue. 

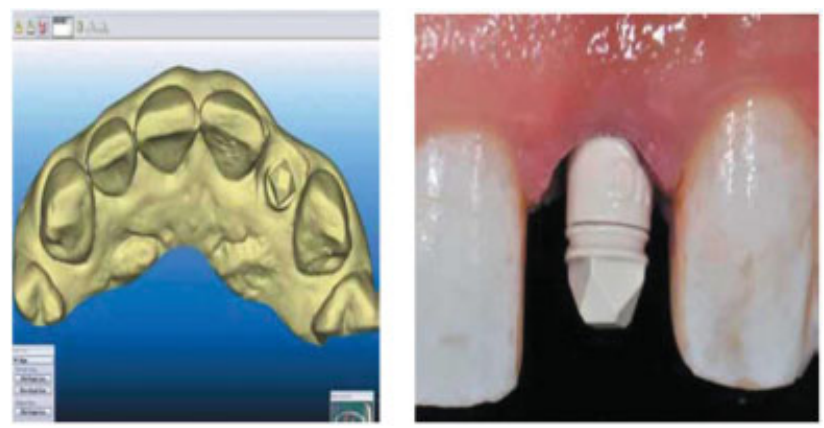

Fig 8 Scannable impression copings.

A digital impression can be created in one of the two ways: via direct intra oral scanning or indirectly via scanning master cast. If scanned directly from the mouth, the digital data can be sent electronically to a milling unit that uses this data to fabricate a digital model; thus, intermediate steps involved in conventional impression making are bypassed. ${ }^{18}$ Digitalization demonstrates two techniques: direct and indirect.

Direct technique: This technique involves making the impression directly in the patients mouth through scanning known as intraoral scanner and a 3D image can be hence easily generated through it. It can then also be saved as a stereolithographic file, which eliminates the need to save the cast as in conventional procedures.

Indirect technique: This technique involves making the impression once the cast has been obtained from the impression made by the open-tray or the closed-tray technique. After pouring the casts in die stone, the cast so obtained is scanned using an extraoral scanner and then the stereolithographic file may be saved and sent for further prosthetic construction.

\section{Infection Control}

Elastomeric impression materials are disinfected by immersing in disinfectant solutions like $2 \%$ glutaraldehyde for 10 minutes. A spray of chlorine dioxide is preferred in case of polyether as it absorbs water. Other disinfectants like phenol and iodophor can also be used.

\section{Conclusion}

The prognosis of implant prosthodontics depends on osseointegration and the passive fit of the prosthesis. Osseointegration depends on precise surgical and restorative procedures, soft tissue management, along with the health of the patient. Any misfit between implant and abutment interface or distortion of framework will not be accommodated like periodontal ligament in natural tooth as implant osseointegration is an ankylosed type of a joint between implant and bone. If there are any discrepancies in the passive fit of the prosthesis, it may lead to screw loosening, screw facture, occlusal interferences, and plaque accumulation, in turn implant failure. Implant impression is one of the most important key factors to get accurate cast, accurate framework with passive fit. Further, the accuracy of cast depends on type of tray, technique, and the material used.
The advent of CAD/CAM technology improved the framework fabrication procedures and has increased the precise fit of implant prosthesis. Any restorative procedure, including implant prosthesis, to get better esthetics and functional results and negative replica of the intended site is the ultimate goal. A good impression making is not just an art but lot of science also.

Conflict of Interest

None identified.

\section{References}

1 Sumathi K, Mantra Sneha S, Deogade Suryakant C. Impressions in implant dentistry - a review. Int J Res Dentistry 2015;5(02):22-32

2 Ravi SY, Sahoo S, Krishna Hari M, Kumar Shameen P, Kumar Satyendra T, Narula S. Accuracy of implant impressions using various impression techniques and impression materials. J Dent Implant 2016;6(01):29-36

3 Inturregui JA, Aquilino SA, Ryther JS, Lund PS. Evaluation of three impression techniques for osseointegrated oral implants. J Prosthet Dent 1993;69(05):503-509

4 Siadat H, Alikhasi M, Beyabanaki E, Rahimian S. Comparison of different impression techniques when using the all on four implant treatment protocol. Int J Prosthodont 2016;29(03):265-270

5 Elshenawy EA, Alam-Eldein AM, Abd Elfatah FA. Cast accuracy obtained from different impression techniques at different implant angulations (in vitro study). Int J Implant Dent 2018;4(01):9

6 Ongül D, Gökçen-Röhlig B, Şermet B, Keskin H. A comparative analysis of the accuracy of different direct impression techniques for multiple implants. Aust Dent J 2012;57(02):184-189

7 Chee W, Jivraj S. Impression techniques for implant dentistry. $\mathrm{Br}$ Dent J 2006;201(07):429-432

8 Lee H, So JS, Hochstedler JL, Ercoli C. The accuracy of implant impressions: a systematic review. J Prosthet Dent 2008;100(04):285-291

9 Daoudi MF, Setchell DJ, Searson LJ. A laboratory investigation of the accuracy of two impression techniques for single-tooth implants. Int J Prosthodont 2001;14(02):152-158

10 Lorenzoni M, Birgit S, Walther A. Comparison of the transfer precision of impression materials for the Frialit-2 system. Int Poster J Dent Med 2002;4(03):140

11 Conrad HJ, Pesun IJ, DeLong R, Hodges JS. Accuracy of two impression techniques with angulated implants. J Prosthet Dent 2007;97(06):349-356

12 Bhakta S, Vere J, Calder I, Patel R. Impressions in implant dentistry. Br Dent J 2011;211(08):361-367

13 Burns J, Palmer R, Howe L, Wilson R. Accuracy of open tray implant impressions: an in vitro comparison of stock versus custom trays. J Prosthet Dent 2003;89(03):250-255

14 Balouch F, Jalalian E, Nikkheslat M, et al. Comparison of the dimensional accuracy between open tray and closed tray implant impression technique in $15^{\circ}$ angled implants. J Dent (Shiraz) 2013;14(03):96-102

15 Toth RW. A tray-less impression technique for complete arch implant-supported immediately loaded provisional and definitive restorations. J Prosthet Dent 2005;94(02):202-203

16 Michalakis KX, Kalpidis CD, Kang K, Hirayama H. A simple impression technique for dental implants placed in close proximity or adverse angulations. J Prosthet Dent 2005;94(03):293-295

17 Lin WS, Harris BT, Morton D. The use of a scannable impression coping and digital impression technique to fabricate a customized anatomic abutment and zirconia restoration in the esthetic zone.J Prosthet Dent 2013;109(03):187-191

18 Güth JF, Keul C, Stimmelmayr M, Beuer F, Edelhoff D. Accuracy of digital models obtained by direct and indirect data capturing. Clin Oral Investig 2013;17(04):1201-1208 